\title{
EDITORIAL
}

\section{Innate immunity and inflammation}

\author{
Tsan Sam Xiao
}

Cellular \& Molecular Immunology (2017) 14, 1-3; doi:10.1038/cmi.2016.45; published online 22 August 2016

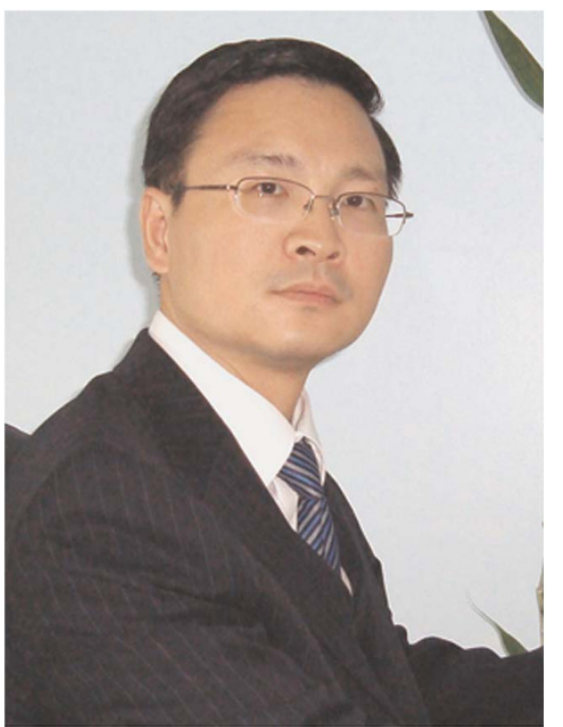

Dr. Tsan Sam Xiao

"But the curative force of nature, the most important element of which is the inflammatory reaction, is not yet perfectly adapted to its object. The frequency of disease and the instances of premature death are a sufficient proof of this." Élie Metchnikoff (1845-1916).

The four cardinal signs of inflammation: rubor (redness), tumor (swelling), calor (heat) and dolor (pain) were first documented by the Roman encyclopedist Aulus Cornelius Celsus in the first century $A D$. The investigation of inflammatory reaction was integrated within the context of protective phagocytosis against noxious

Department of Pathology, Case Western Reserve University, Cleveland, OH 44106, USA

E-mail: tsx@case.edu

Received: 15 July 2016; Accepted: 15 July 2016 agents by Élie Metchnikoff, father of innate and cellular immunity, who elaborated on the function of phagocytes in his lectures on 'Comparative Pathology of Inflammation'. ${ }^{1}$ It is now recognized that inflammation induced by microbial infection and tissue damage is an essential mechanism of innate immune response. Proper inflammatory responses provide broad spectrum protection against infections and orchestrate long-term adaptive immunity toward specific pathogens. In contrast, chronic and uncontrolled inflammation often inflicts severe tissue damage resulting in major pathogenicity from overzealous immune responses. As such, layers of regulatory mechanisms have evolved to fine-tune the initiation, progression and resolution of inflammation elicited by the host immune systems. This special issue of Cellular \& Molecular Immunology assembles six reviews and four research articles that cover various aspects of innate immune responses and inflammation at the molecular, cellular and organismal levels, with special emphasis on regulatory mechanisms derived from host and invading microbes. This issue features recent insights on hostmicrobial interactions on viral, mycobacterial and parasitic infections, and the subversive mechanisms and countermeasures evolved by pathogens and hosts, respectively. Because the activation of pattern recognition receptors and production of effector molecules are essential components of the innate immune system, regulatory mechanisms are examined and discussed for the Toll-like receptors (TLRs), the NLRP3 and AIM2 inflammasomes, and a group of alarmins endowed with both intracellular and extracellular functions.

Co-evolution of host and pathogens often results in immunoevasive strategies adopted by the pathogens that disarm the host defense mechanisms. Christensen and Paludan ${ }^{2}$ discuss some of these subversive strategies that target the type I interferon responses. These include viral ubiquitination enzymes and proteases that promote degradation of host receptors, virus-encoded binding proteins that prevent receptor signaling and gene expression, and viral recruitment of host proteins to prevent immune recognition of viral genome. To adapt to these, host cells developed countermeasures such as bystander activation to bypasses immune evasion, which is discussed by Holmgren et al. ${ }^{3}$ Through direct cellular contacts or paracrine signaling, host cells that harbor pathogens can stimulate inflammatory responses from uninfected cells, thus counteract immune subversion within the infected cells. Using examples of infections by viral, bacterial and protozoan pathogens in fly and human, the authors argue that bystander activation is a crucial evolutionary adaptation in metazoan immunity. Continuing the theme of inflammation on viral infections, Ireland et al. ${ }^{4}$ demonstrate that infection of neonatal mice by Tacaribe virus leads to viral accumulation in the brain stem and cerebellum, accompanied by lethal meningoencephalitis. Such neuropathology is dependent on the infiltration of $\mathrm{T}$ cells and monocytes in the brain following viral infection. However, antigen-specific 
$\mathrm{T}$ cells not only fail to clear the virus but also exacerbate neuropathology from excessive host inflammatory responses against Tacaribe virus infection, with the $\mathrm{CD} 4+$ and $\mathrm{CD} 8+\mathrm{T}$ cells play distinct roles. This suggests that therapies that modulate $\mathrm{T}$ cell responses to viral infections in the central nervous system may need to tailor toward specific cell types. Furthermore, attempts to employ $\mathrm{T}$ cells as immunotherapies need to be carefully evaluated to avoid severe neuropathology. The role of inflammatory cytokines upon infection by Mycobacteria tuberculosis is explored by Mayer-Barber and Yan. ${ }^{5}$ They discuss the counterregulation of two crucial innate cytokines, type I interferon and interleukin-1, and the distinct inflammatory responses each initiates in the context of mycobacterial infection. The intracellular parasite Toxoplasma gondii infects various mammals including mouse, which has been used extensively to model human toxoplasma infection. Sher et al. ${ }^{6}$ summarize recent findings that human myeloid subsets initiate different cytokine responses to $T$. gondii infection compared with their mouse counterparts. Although TLRs are essential for sensing of soluble profilin from the parasite by murine myeloid cells, the human immune responses to $T$ gondii appear to rely on phagocytic uptake of live parasite and may employ cytosolic nucleic acid sensors. Such distinct innate recognition of $T$. gondii by human and murine myeloid cells may be relevant to their different roles in the life cycle of the parasite, and suggests careful evaluation of model organisms as surrogates for human toxoplasma study.

Pattern recognition receptors, first proposed by Charles Janeway, ${ }^{7}$ are essential molecular sentinels of the innate immune system that recognize conserved microbial components and elicit the production of proinflammatory effector molecules. The TLRs are prototype pattern recognition receptors that sense a variety of microbial epitopes. Tomalka et al. ${ }^{8}$ report the modulation of TLR-induced NF-kB and mitogen-activated protein kinase (MAPK) activation by a novel regulator Sam68. Interestingly, the TLR2, TLR3 and TLR4 pathways have differential requirement of Sam68 for the activation of NF-kB c-Rel or p65, while Sam68 negatively regulates MAPK activation. Even though the detailed molecular mechanisms of such regulation by Sam68 remain to be elucidated, these data suggest that Sam68 may function as an immune rheostat that regulates balanced activation of both NF-kB and MAPK pathways. In addition to microorganisms, misplaced or altered host molecules, or alarmins, can also stimulate pattern recognition receptors following tissue damage. Bertheloot and Latz ${ }^{9}$ review recent findings on sterile inflammation induced by HMGB1, IL-1, IL-33 and S100 proteins. As these molecules play important roles in both the promotion of tissue repair and the exacerbation of inflammatory disorders, targeting their regulatory mechanisms may offer potential therapeutic opportunities for a number of diseases including cancer.

Inflammasomes constitute a group of pattern recognition receptors capable of assembling large oligomeric signaling complexes that promote the activation of caspases and secretion of proinflammatory cytokines. ${ }^{10}$ Because inflammasomes respond to both sterile and microbial stimulation, Bezbradica et al. ${ }^{11}$ investigate the differential activation of the NLRP3 inflammasome by these two categories of stimulation. Their study reveals that the microbial stimulation induces faster and more robust NLRP3 activation in both human and mouse macrophages, perhaps reflecting the need for swift containment on pathogen infection. This suggests that distinct regulatory mechanisms triggered by microbial or sterile stimulation dictate differential activation of the NLRP3 inflammasome, and more quantitative analysis of the activation process of other inflammasomes may be warranted. One of these is the AIM2 inflammasome examined by Ratsimandresy et al. ${ }^{12}$ in mucosal immunity. The activation of inflammasomes in hematopoietic cells is known to exacerbate intestinal inflammation, while those in intestinal epithelial cells promote the production of IL-18 essential for tissue regeneration and reduced colorectal cancer incidence. The AIM2 receptor in nonhematopoietic cells was shown to protect from colorectal cancer through DNAdependent protein kinase and Akt pathways, independent of the assembly of the AIM2 inflammasome. ${ }^{13,14}$ Here Ratsimandresy and colleagues report that the AIM2 inflammasome in intestinal epithelial cells does play an essential role in preventing dysbiosis-mediated intestinal inflammation. Mechanistically the fecal microbiota DNA activates the AIM2 inflammasome and stimulates the production of IL-18. This in turn regulates the expression of select antimicrobial peptides of the Reg3 family through the IL-18/IL-22/STAT3 axis, which restore tissue barrier and homeostasis of the intestinal microbiota. It is clear that inflammasome activities are regulated in a cell-type and stimulationdependent manner. Some of the regulatory mechanisms for inflammasomes are reviewed by Yang et al. ${ }^{15}$ in the context of post-translational modifications. Because highly diverse and versatile post-translational modifications shape distinct inflammatory responses, enzymes involved in these modifications may become novel therapeutic targets.

In summary, this special issue of CMI features discussion and update that address many facets of innate immunity and inflammatory responses. A common theme that emerges from these is that regulatory mechanisms derived from host and microorganisms can modulate inflammatory responses by different signaling pathways in distinct cell types. Accumulating insights into the intricacies of these regulatory mechanisms may lead to better understanding of the pathogenesis of various diseases and tailored therapeutic strategies against them.

1 Metchnikoff E. Lectures On The Comparative Pathology of Inflammation: Delivered at the Pasteur Institute in 1891. Kegan Paul, Trench, Trubner \& Co., LTD. 1893.

2 Christensen MH, Paludan SR. Viral evasion of DNA-stimulated innate immune responses. Cell Mol Immunol 2016; e-pub ahead of print 14 March 2016; doi:10.1038/cmi.2016.06.

3 Holmgren AM, McConkey CA, Shin S. Outrunning the red queen: bystander activation as a means of outpacing innate immune subversion by intracellular pathogens. Cell Mol Immunol 2016; 13: in press. 
4 Ireland DDC, Tami C, Pedras-Vasconcelos J. CD4 Verthelyi D. and CD8 $T$ cells mediate distinct lethal meningoencephalitis in mice challenged with Tacaribe arenavirus. Cell Mol Immunol 2016; 13: in press.

5 Mayer-Barber K, Yan B. Clash of the cytokine titans: counter-regulation of interleukin 1 and type I interferon mediated inflammatory responses. Cell Mol Immunol 2016; e-pub ahead of print 6 June 2016; doi:10.1038/ cmi.2016.25.

6 Sher A, Tosh K, Jankovic D. Innate recognition of Toxoplasma gondii in humans involves a mechanism distinct from that utilized by rodents. Cell Mol Immunol 2016; e-pub ahead of print 9 May 2016; doi:10.1038/ cmi.2016.12.

7 Janeway CA. Approaching the Asymptote? Evolution and Revolution in Immunology.
Cold Spring Harb Symp Quant Biol 1989; 54: 1-13.

8 Tomalka JA, de Jesus TJ, Pamakrishnan P. Sam68 is a regulator of Toll like receptor signaling. Cell Mol Immunol 2016; e-pub ahead of print 4 July 2016; doi:10.1038/ cmi.2016.32.

9 Bertheloot D, Latz E. HMGB1, IL1, IL-33 and S100 proteins; dual-function alarmins. Cell Mol Immunol 2016; 13: in press.

10 Martinon F, Burns K, Tschopp J. The inflammasome: a molecular platform triggering activation of inflammatory caspases and processing of prolL-beta. Mol Cell 2002; 10: 417-426.

11 Bezbradice JS, Coll RC, Schroder K. Sterile signals generate weaker and delayed macrophage NLRP3 inflammasome responses relative to microbial signals. Cell Mol Immunol
2016; e-pub ahead of print 21 March 2016; doi:10.1038/cmi.2016.11.

12 Ratsimandresy RA, Indramohan M, Dorfleutner A, Stehlik C. The AIM2 inflammasome is a central regulator of intestinal homeostasis through the IL-18/IL-22/STAT2 pathway. Cell Mol Immunol 2016; 13: 1-17.

13 Wilson JE, Petrucelli AS, Chen L, Koblansky AA, Truax AD, Oyama Y et al. Inflammasomeindependent role of AIM2 in suppressing colon tumorigenesis via DNA-PK and Akt. Nat Med 2015; 21: 906-913.

14 Man SM, Zhu Q, Zhu L, Liu Z, Karki R, Malik A et al. Critical role for the DNA sensor AIM2 in stem cell proliferation and cancer. Cell 2015; 162: 45-58.

15 Yang J, Liu Z, Xiao TS. Post-translational regulation of inflammasomes. Cell $\mathrm{Mol}$ Immunol 2016; e-pub ahead of print 27 June 2016; doi:10.1038/cmi.2016.29. 